\section{Re: Magnesium Intake and Depression in Adults}

To the Editor: We read with great interest the article by Tarleton and Littenberg ${ }^{1}$ on the relation between magnesium intake and depression in adults. We recognize the impact of depression in modern society, and we agree with the authors that current treatment options have their limitations. A possible role for magnesium could therefore be interesting. The authors found an association between low magnesium intake and depression among younger adults and a potential protective effect against depression in seniors. Although this sounds promising, we would like to discuss some methodologic issues about this study.

First, the authors chose to divide the range of magnesium intake within the study population into quantiles. This is remarkable, as the quintiles do not correspond to magnesium intake thresholds as defined by the estimated average requirements. ${ }^{2}$ The reason for the use of quantiles is not explained but would be of interest for the readers, since this choice has important consequences on the outcome: When the estimated average requirement definition for deficient magnesium intake is used, no association between magnesium intake and depression was found (adjusted relative risk $0.98 ; 95 \%$ confidence interval [CI], 0.88-1.07).

Second, based on 1-day dietary recall data, Tarleton and Littenberg ${ }^{1}$ found a deficient magnesium intake in $54 \%$ of the study population. Although this is a relatively high percentage, the authors stated in their discussion that the method of magnesium assessment was adequate. However, it is important to realize that the absorption of magnesium varies depending on the intake of magnesium. The absorption can vary between $30 \%$ and $40 \%$ in a normal diet and up to $80 \%$ with a low magnesium intake. ${ }^{3}$ Therefore, deficient magnesium intake cannot directly be translated into actual hypomagnesemia. From this perspective it is questionable whether the authors are really evaluating the magnesium status of their study group.

Third, when evaluating the multivariable adjusted analyses of low magnesium and depression, it is obvious that most variables have a much stronger association with depression than low magnesium intake (at most chronic kidney disease: odds ratio [OR], 2.50; 95\% CI, 1.663.79; and food insecurity: OR, 2.30; 95\% CI, 1.90-2.78). All those variables seem to be associated with socioeconomic status (SES) and lifestyle. Low magnesium intake may therefore be a proxy for an unhealthy lifestyle and/or a low SES. The association between SES and depression is well known: Low SES increases the risk of onset and persistence of depression. ${ }^{4}$ In addition, depression has been found to be associated with poor food consumption patterns, with the association probably being bidirectional. ${ }^{5,6}$

In conclusion, the idea that magnesium intake could modulate depression is interesting. Given the abovementioned concerns, however, we are doubtful about the described association between magnesium intake and depression. When further research is considered, we recommend first a prospective study instead of a randomized clinical trial.

Denise Sluimers, MS*

Nick L. Willemse, $\mathrm{MS}^{*}$

Marieke L.A. Landsmeer

Department of General Practice

Erasmus Medical Center

Rotterdam, The Netherlands

m.landsmeer@erasmusmc.nl

*These authors contributed equally to this letter.

\section{References}

1. Tarleton EK, Littenberg B. Magnesium intake and depression in adults. J Am Board Fam Med 2015;28:249-56.

2. Institute of Medicine (US) Standing Committee on the Scientific Evaluation of Dietary Reference Intakes. Dietary Reference Intakes for Calcium, Phosphorus, Magnesium, Vitamin D, and Fluoride. Washington, DC: National Academies Press; 1997. Available from: http://www.ncbi.nlm.nih.gov/ books/NBK109825/. Accessed July 27, 2015.

3. McLean RM. Magnesium and its therapeutic uses: a review. Am J Med 1994;96:63-76.

4. Lorant V, Croux C, Weich S, Deliege D, Mackenbach J, Ansseau M. Depression and socio-economic risk factors: 7-year longitudinal population study. Br J Psychiatry 2007; 190:293-8.

5. Akbaraly TN, Brunner EJ, Ferrie JE, Marmot MG, Kivimaki M, Singh-Manoux A. Dietary pattern and depressive symptoms in middle age. Br J Psychiatry 2009;195:408-13.

6. Anton SD, Miller PM. Do negative emotions predict alcohol consumption, saturated fat intake, and physical activity in older adults? Behav Modif 2005;29:677-88.

doi: 10.3122/jabfm.2015.05.150144

The above letter was referred to the author of the article in question, who offers the following reply.

\section{Response: Re: Magnesium Intake and Depression in Adults}

To the Editor: We thank Slumiers and colleagues ${ }^{1}$ for highlighting the challenges of interpreting outcomes from cross-sectional, population-based data. Preclinical and clinical studies indicate low magnesium intake may be associated with depressive symptoms. For instance, Singewald et $\mathrm{al}^{2}$ reported mice consuming a diet with very low magnesium content-consisting of only $10 \%$ of 